\title{
THE MISSING GOLIATH'S SLINGSHOT: MASSIVE BLACK HOLE RECOIL AT M83
}

\author{
Horacio Dottori ${ }^{1}$, Rubén J. Díaz ${ }^{2}$, Juan Facundo Albacete-Colombo ${ }^{3}$, And Damián Mast ${ }^{4,5}$ \\ ${ }^{1}$ Instituto de Física, Universidade Federal do Rio Grande do Sul, Porto Alegre, Brazil; dottori@if.ufrgs.br \\ ${ }^{2}$ Gemini Observatory, AURA, Colina El Pino, La Serena, Chile; rdiaz@ gemini.edu \\ ${ }^{3}$ Universidad Nacional del Comahue (CURZA), M. Esandi y Ayacucho S/N, Viedma CP 8500, Rio Negro, Argentina \\ ${ }^{4}$ Centro Astronómico Hispano Alemán, Calar Alto, (CSIC-MPG), Almería, Spain \\ ${ }^{5}$ Instituto de Astrofísica de Andalucía-CSIC, C/Bajo de Huétor, 50, 18008 Granada, Spain \\ Received 2009 October 7; accepted 2010 May 13; published 2010 June 14
}

\begin{abstract}
The Fanaroff-Riley II radio source J133658.3-295105, which is also an X-ray source, appears to be projected onto the disk of the barred-spiral galaxy M83 at about 60" from the galaxy's optical nucleus. J133658.3-295105 and its radio lobes are aligned with the optical nucleus of M 83 and two other radio sources, neither of which are supernova remnants or H II regions. Due to this peculiar on-the-sky projection, J133658.3-295105 was previously studied by Gemini+GMOS optical spectroscopy, which marginally revealed the presence of $\mathrm{H} \alpha$ in emission receding at $130 \mathrm{~km} \mathrm{~s}^{-1}$ with respect to the optical nucleus. In this Letter, we reanalyze the Chandra spectroscopy carried out in 2000. We show that J133658.3-295105 presents an Fe K $\alpha$ emission line at a redshift of $z=0.018$. This redshift is compatible with a black hole at the distance of $M$ 83. We discuss similarities to the recently reported micro-quasar in NGC 5408. This finding reinforces the kicked-off black hole scenario for J133658.3-295105.
\end{abstract}

Key words: galaxies: evolution - galaxies: individual (M 83) - galaxies: kinematics and dynamics - galaxies: nuclei - X-rays: individual (J 133658.3-295105)

\section{INTRODUCTION}

The correlation between supermassive black hole $(\mathrm{SMBH})$ mass and bulge velocity dispersion in spiral galaxies (Ferrarese $\&$ Merrit 2000; Gebhardt et al. 2000) points to a strong interplay between these systems in a hierarchical scenario of disk-galaxy formation (Okamoto et al. 2008). A paradigmatic outcome of a merger of $\mathrm{BHs}$ is the recoil of the resulting $\mathrm{BH}$ (Bonning et al. 2007; Blecha \& Loeb 2006) in response to the anisotropic emission of gravitational waves (Blecha \& Loeb 2006). In a triple $\mathrm{BH}$ system, the ejection of the smallest $\mathrm{BH}$ may happen far in advance of the merger of the two largest objects (Iwasawa et al. 2006). There are two caveats to this scenario: the search for kicked-off BHs has been unsuccessful thus far (Bogdanovic et al. 2009), and supermassive binary BH systems, which should also be common objects, are elusive (Boroson \& Lauer 2009).

The source J133658.3-295105 emits X-rays (Soria \& Wu 2003; XS39, sources from Soria \& Wu's list will be cited with the list number preceded by XS) and is also resolved in radio (Maddox et al. 2006; RS28, sources from Maddox et al. will be cited with the list number preceded by RS). RS28 is characterized as a Fanaroff-Riley type II (FRII) radio source. In addition to a flat-spectrum radio core, RS28 also presents two distinct radio lobes clearly showing the effect of relativistic boosting at $25^{\prime \prime} \sim 0.5 \mathrm{kpc}$ northwest (RS27) and southeast (RS29) of the core. They are projected onto the disk of the barred spiral M83 at about $60^{\prime \prime}(\sim 1.3 \mathrm{kpc})$ of the galaxy's optical nucleus (ON). J133658.3-295105 and the radio lobes present an on-the-sky alignment with respect to the galaxy ON (Cowan et al. 1994) and the radio sources RS30 and RS32 (Dottori et al. 2008a, 2008b). The ON of M83 itself is shifted from the galaxy's kinematic center (KC; Thatte et al. 2000). Merger processes (Diaz et al. 2006), as well as galactic inner buildup (Elmegreen et al. 1998), are now evident in M83. At a distance of about 4.5 Mpc (Karachentsev et al. 2002), M83 belongs to the Hydra-Centaurus group, one of the most active regions in the nearby universe and recently detected as a source of ultra high-energy cosmic rays (Abraham 2007). Soria \& Wu (2003) proposed a distance of $z \geqslant 1$, arguing that known FRII radio galaxies always have a lower limit of $L_{6 \mathrm{~cm}}$ and $L_{20 \mathrm{~cm}}$ (Kembhavi \& Narlikar 1999). Gemini+GMOS observations (Dottori et al. 2008b; GS-2007A-DD-17) marginally detected $\mathrm{H} \alpha$ emission coming from J133658.3-295105. This emission indicates a receding speed of $130 \pm 40 \mathrm{~km} \mathrm{~s}^{-1}$ with respect to the M83 ON. In this study, we critically analyze the detection of Fe $\mathrm{K} \alpha \mathrm{X}$-ray emission line in the X-ray counterpart of J133658.3-295105 (XS39).

\section{CHANDRA X-RAY OBSERVATION}

Of the three available X-ray observations of the M83 spiral galaxy (one from XMM-Newton and two from Chandra), XS39 was detected in the deepest Chandra observation on 2000 April 29 (ObsID: 793). It is located $\sim 1^{\prime \prime}$ off-axis from the ACIS-S3 aim point. The total exposure time was $50.981 \mathrm{ks}$. After screening out observational intervals with strong background flares, a good time interval of $50.811 \mathrm{ks}$ was retained. We hereafter assume a non-variable background. Data were acquired in the FAINT observation and TIMED read modes. Data reduction, starting with the Level 1 event list provided by the pipeline processing at the Chandra X-Ray Observatory, was performed using the latest version of $\mathrm{CIAO}^{6}$ (ver. 4.1.2) and the CALDB (ver. 4.1.3) set of calibration files. Photon energies were corrected for the time dependence of the energy gain using the CORR_TGAIN CIAO task. To avoid calibration uncertainties, the event data were filtered on energy using the range $0.5-8.0 \mathrm{keV}$.

In Figure 1, we show the projected position of XS39 on the disk of M83 in a false color combination of three X-ray bandpasses plus a single $\mathrm{H} \alpha$ Gemini image. Before the colorcoded image combination, we corrected the single-band X-ray images for variations in exposures, sensitivity, and vignetting by computing and dividing with the respective exposure maps.

\footnotetext{
6 http://cxc.harvard.edu/ciao
} 


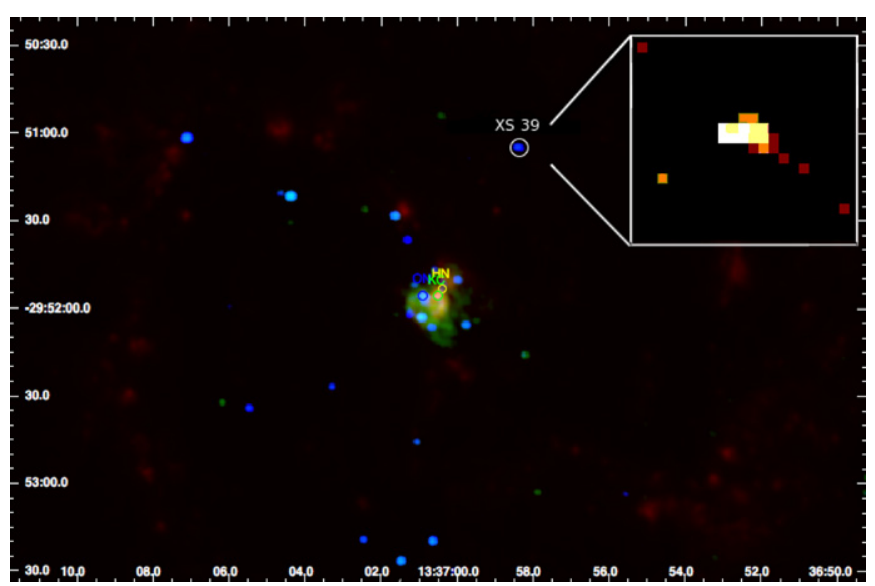

Figure 1. False four-color image of M83. The Chandra X-ray frames are composed of yellow $(0.5-1.2 \mathrm{keV})$, green $(1.2-2.5 \mathrm{keV})$, and blue $(2.5-8.0 \mathrm{keV})$ energies. The red image corresponds to the $\mathrm{H} \alpha$ image from the Gemini south telescope. The Chandra ACIS-S image has been smoothed, and pixel intensities are displayed in a logarithmic scale. The inner panel of the figure shows that the X-ray emission of XS39 appears to be slightly elongated due to the local PSF of ACIS at the $\sim 1^{\prime \prime}$ off-axis angle. Note: the $\mathrm{KC}, \mathrm{ON}$, and hidden nucleus (HN) are indicated near the M83 core.

The hardness of the X-rays from XS39 is clear from Figure 1, indicating that most of the emission is in the $2-8 \mathrm{keV}$ band.

\subsection{X-ray Spectrum of J133658.3-295105}

The spectral data extraction was performed by following the standard CIAO thread to extract an ACIS spectrum. The steps were as follows: (1) extract source events within an ellipse of semi-axes $2^{\prime \prime} .2$ and $1^{\prime \prime} .6$ and a position angle of $150^{\circ}$, mapping the elongated local point-spread function (PSF) of the ACIS-S camera at $\sim 1^{\prime}$ (see Figure 1, inner panel); (2) create the aspect histogram file; and (3) create the RMF and ARF files that are appropriate for the time-dependent source position on the chip. In addition to the standard thread, the event data were filtered based on energy using the range $0.5-8.0 \mathrm{keV}$. The resulting net detection of photons was 135 . There was a net count rate of $2.6 \times 10^{-3}$ counts $\mathrm{s}^{-1}$. To improve the signal-to-noise ratio $(\mathrm{S} / \mathrm{N})$ of the spectrum while avoiding excessive spectral resolution degradation, we binned the spectra to a minimum of two photons per bin. Spectral fitting on the ACIS data was carried out with the Interactive Spectral Interpretation System (ISIS; Houck $\&$ Denicola 2000). Because of the low photon statistic in the $\mathrm{X}$-ray spectrum, the $\chi^{2}$ statistic was avoided. Instead, we used the SIMPLEX minimization method and the Cash statistic (Cash 1979).

By using the $C$-stat statistic and a pure blackbody or a single power-law model to fit the X-ray spectrum of XS39, we found that the residual contribution in the $C$-statistic regime, at energies of the $\mathrm{Fe} \mathrm{K} \alpha(6.7 \mathrm{keV})$ line complex, is around a factor of 40 or larger. This suggests that simple models are clearly unrealistic in describing the X-ray spectrum of XS 39.

The XS39 X-ray spectrum (see Figure 2) is probably inconsistent with a pure blackbody or single power law. In the minimization procedure, we use a physical model that assumes the presence of a thermal plasma in collision-filled ionization equilibrium, as modeled by the APEC code of Brickhouse et al. (2005). A Gaussian emission line is also assumed to fit the $\mathrm{Fe}$ $\mathrm{K} \alpha$ complex (see Figure 2 for details).

The statistical significance of the $\mathrm{Fe} \mathrm{K} \alpha$ line in a photonlimited spectrum is difficult to determine, even more so if it has seven photons over the continuum, as in the case of the X-ray
Table 1

Spectral Fit Parameters

\begin{tabular}{lc}
\hline \hline Model Parameters & APEC+GAUSS \\
\hline apec & $0.96 \pm 0.3$ \\
$N_{\mathrm{h}}\left(10^{22} \mathrm{~cm}^{-2}\right)$ & $7.7 \pm 1.9$ \\
$k T(\mathrm{keV})$ & $3.0 \times 10^{-5}$ \\
Normalization & 1.0 \\
Abundance & \\
\hline Fe-K line & $6.52 \pm 0.04$ \\
Peak & $6.5 \times 10^{-7}$ \\
Normalization & $\cdots$ \\
$R$ (in-out) & $\cdots$ \\
Inclination (deg) & $0.018_{-0.006}^{+0.006}$ \\
Redshift & $4 \times 10^{-15}$ \\
Flux $_{\mathrm{FeK}}(\mathrm{cgs})$ & 0.69 \\
$C_{v}$-stat & $3.5 \times 10^{-14}$ \\
Flux $_{\mathrm{x}}(\mathrm{cgs})$ & $5.6 \times 10^{37}$ \\
$L_{\mathrm{x}}\left(\mathrm{erg} \mathrm{s}{ }^{-1}\right)$ & \\
\hline
\end{tabular}

Notes. The model was multiplicatively affected by Galactic absorption $N_{\mathrm{H}}=4.0 \times 10^{20} \mathrm{~cm}^{-2}$. Model normalization is in units of photons $\mathrm{s}^{-1} \mathrm{~cm}^{-2} \mathrm{keV}^{-1}$. X-ray flux, in units of $\mathrm{erg} \mathrm{s}^{-1} \mathrm{~cm}^{-2}$, was computed in the $0.5-8 \mathrm{keV}$ energy range. Ninety percent confidence limits were computed and expressed as error in the table. Note that by using a two-thermal emission model, we also fit the $\mathrm{X}$-ray spectrum of the optical nucleus (ON). The computed X-ray flux and luminosity of the ON were $1.6 \times$ $10^{-13} \mathrm{erg} \mathrm{s}^{-1} \mathrm{~cm}^{-2}$ and $2.62 \times 10^{38} \mathrm{erg} \mathrm{s}^{-1}$, respectively. The $\mathrm{Fe} \mathrm{K} \alpha$ line did not appear in the ON X-ray spectrum.

spectrum of XS39. However, we can obtain an estimation by a direct comparison of the Fe-K line $\chi^{2}$ residuals (see Figure 2, right panel). We study the residuals at the $6.7 \mathrm{keV}$ energy by computing the $C$-stat residuals of a thermal (APEC + GAUSSIAN) model that includes the $\mathrm{K} \alpha$ line with respect to a single APEC model. We found that local residuals are about 0.3 and $\sim 7$, respectively. This implies that the inclusion of a Gaussian line at the $6.7 \mathrm{keV}$ energy yields residuals that are 20 times smaller. A robust result in terms of the significance of the $\mathrm{Fe} \mathrm{K} \alpha$ line cannot be determined accurately because of the statistical comparison between these two distributions. Otherwise, we were able to compute the statistical confidence ranges of the emission line by using the normalization parameter of the Gaussian fit. As shown in Table 1, this line accounts for about $9 \%$ of the total $\mathrm{X}$-ray flux, i.e., $4 \times 10^{-15} \mathrm{erg} \mathrm{s}^{-1} \mathrm{~cm}^{-2}$. The confidence levels of the line can be computed from the Gaussian line normalization, i.e., GAUSSSIAN.NORM $\left(6.5 \times 10^{-7}\right)$ parameter. We use the ISIS CONF task to compute the $90 \%$ confidence limit of the $\mathrm{Fe} \mathrm{K} \alpha$ line amplitude at $\sim 1.3 \times 10^{-6}-3.6 \times 10^{-7}$, which covers a factor of $50 \%$ in flux. However, the $99 \%$ confidence limit ranges between $1.5 \times 10^{-6}$ and $8.7 \times 10^{-8}$, which is almost an order of magnitude below the best-fit value. Hence, the fitted Gaussian $\mathrm{Fe} \mathrm{K} \alpha$ line is $\sim 2.5 \sigma$ over the continuum level.

\section{RESULTS}

The models in Table 1 give a redshift of $z=0.018_{-0.007}^{+0.006}$. Within a $2 \sigma-3 \sigma$ accuracy level of this determination, it is found to be compatible with the receding velocity of $130 \mathrm{~km} \mathrm{~s}^{-1}$ found from the $\mathrm{H} \alpha$ emission line (Dottori et al. 2008b) and with the currently computed velocities of a kick-off (Blecha \& Loeb 2006). We remark that the $\mathrm{Fe} \mathrm{K} \alpha(6.7 \mathrm{keV})$ clearly indicates $z \ll 1$. 

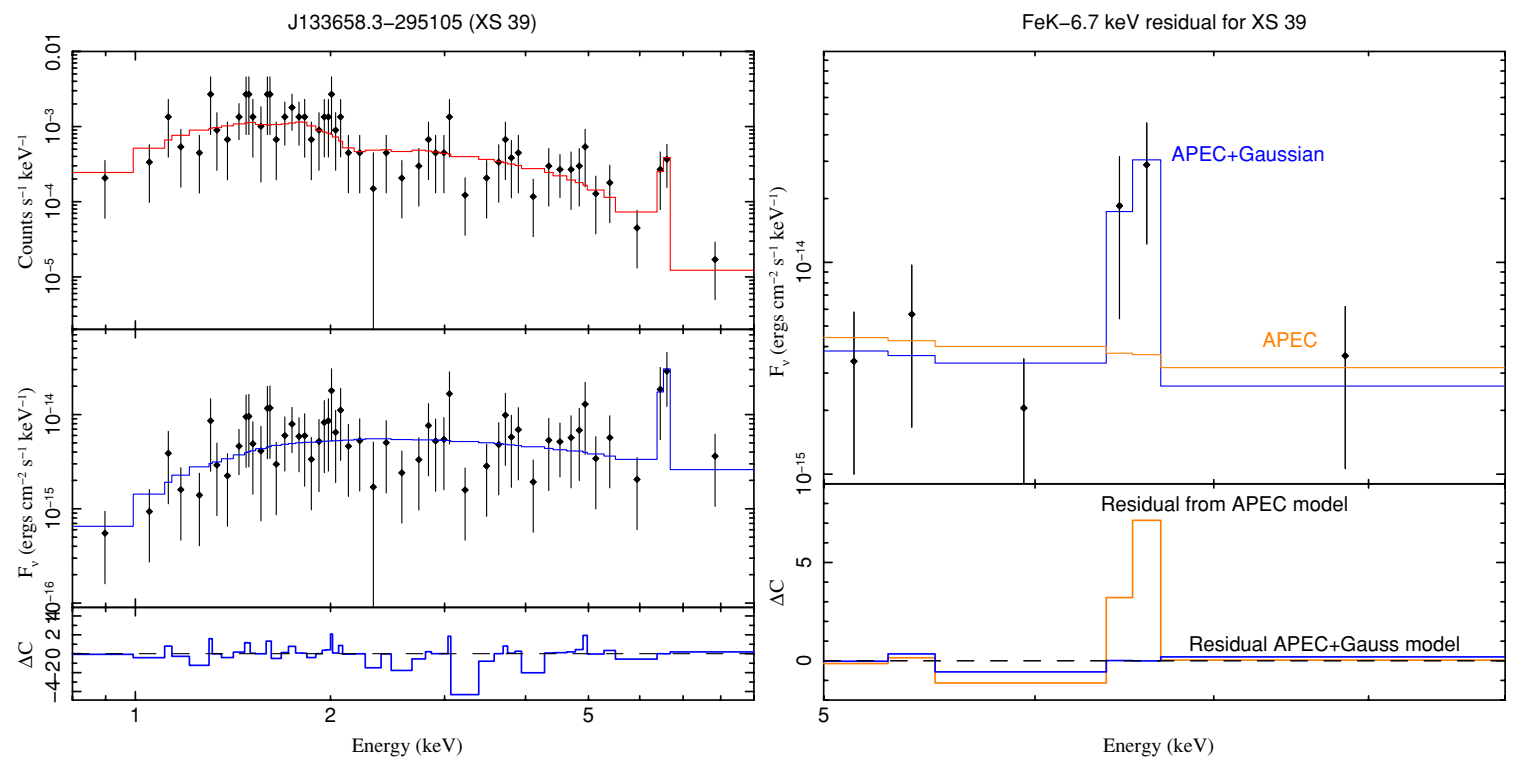

Figure 2. Left: spectral photon energy distribution of the XS39 (upper panel). Unfolded X-ray spectrum appears in the middle panel. The spectrum is binned to a minimum of two photons per bin. $C$-statistic fit residuals appear in the bottom panel. Right: $C$-statistic residuals of the Fe $\mathrm{K} \alpha$ line $(6.7 \mathrm{keV})$ for a model that includes a Gaussian line plus a thermal emission (APEC) or a model without line emission.

Figure 3 shows a blown-up portion of the Figure 1 field, along a line through the $\mathrm{ON}$ and XS39, in which the X-ray sources are superimposed on a radio image. The astrometry of the maximum brightness of XS39 and RS28 is shown in two-dimensional coordinates elsewhere (Dottori et al. 2008b, Figure 3). $(\alpha, \delta)$ agrees within $0.5 \sim 10 \mathrm{pc}$ with the previous determinations for the two objects, namely, $(13: 36: 58.39,-29: 51: 05.1)$ for XS39 (Soria \& Wu 2003) and (13:36:58.34, -29:51:04.58) for RS28 (Maddox et al. 2006). The X-ray spectrum of XS39 is unique (Soria \& Wu 2003) among the other 127 X-ray sources found within an area of $12^{\prime} \times 12^{\prime}$ centered at the nucleus of M83. A circle with a radius of 0.5 is the upper limit for the superposition area between the RS28 and XS39 position error circles. Therefore, an alignment by chance of a putative background radio galaxy (RS28) with XS39 will be proportional to the area of this circle divided by the area covered by the Chandra frame, which means $\approx 1: 6 \times 10^{5}$. That makes the physical association of RS28 and XS39 highly feasible, as noted by different authors (Stockdale et al. 2001; Soria \& Wu 2003; Maddox et al. 2006).

At the distance of M83, the projected distance between the radio lobes of J133658.3-295105 is about $1 \mathrm{kpc}$. As a matter of comparison, the distance between lobes in FRII radio sources, centered in nuclei of active galaxies and quasars $\left(\mathrm{DRAGN}^{7}\right)$ covers a wide range of lengths, from a few tens of pc to several Mpc. There is nothing odd with the double-source separation, but only with the location of the central source coming outward from the nuclear region. From this point of view, off-centered micro-quasars and the Ultra-Luminous X-Ray Source (ULX) observed in the Milky Way and nearby galaxies are more adequate reference objects. The recently detected largest extragalactic micro-quasar in NGC7793 has a lobe-tolobe projected size of more than $\approx 250 \mathrm{pc}$ (Soria et al. 2009). The authors consider this object as an analog of SS433. Contrary to these micro-quasars, J133658.3-295105 does not show signs of a surrounding nebula, as SS433 does (W50 nebula), suggesting that J133658.3-295105 is outside of the galactic

\footnotetext{
7 www.jb.man.ac.uk/atlas/index.html
}

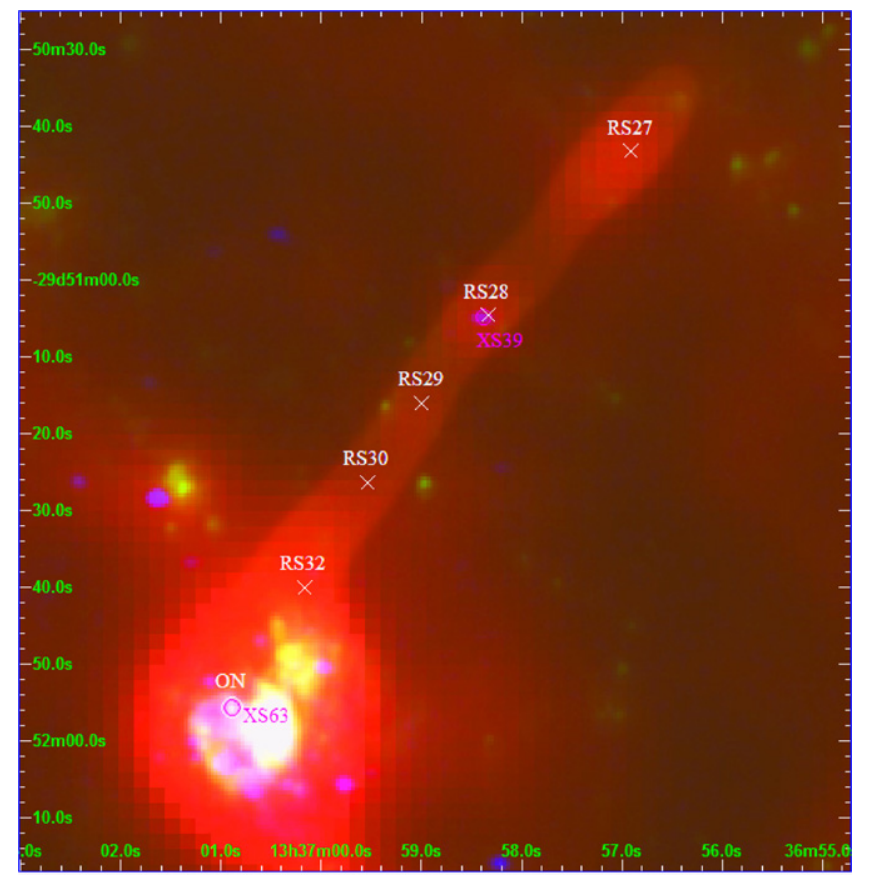

Figure 3. Blow-up along the line through the optical nucleus and XS39. The $\mathrm{X}$-ray sources are shown in blue-white, the $\mathrm{H} \alpha$ emission in green, and the $6 \mathrm{~cm}$ radio emission in red. We detached the maxima of the radio sources along the jet-like radio feature as well as the ON. XS39 and RS28 are the X-ray and radio identifiers of J133658.3-295105, respectively.

plane. This scenario is supported by the redshift derived from the $\mathrm{H} \alpha$ emission line (Dottori et al. 2008b).

If J133658.3-295105 were similar to the active galactic nuclei (AGNs), the ratio $\log (F(X) / F(\mathrm{opt}))$ would be about 1 (Mushotzky 2004). The X-ray flux quoted in Table 1 is $F(0.2-8.0 \mathrm{keV}) \approx 3.5 \times 10^{-14} \mathrm{erg} \mathrm{cm}^{-2} \mathrm{~s}^{-1}$, which would correspond to an optical counterpart at the 18-19 mag level. This is at least 3 mag brighter than the detection limit of the available GMOS/Gemini-S imagery (Dottori et al. 2008b), which does not show such a bright optical counterpart. Indeed, from a direct 
comparison with the observations, the authors estimate that their composed image could detect more than $80 \%$ of the broad and narrow line AGNs at $1 \leqslant z \leqslant 3$ in the SEXSI sample (Eckart et al. 2006), irrespective of the redshift. In agreement with Soria \& Wu (2003) work, our analysis does not show X-ray variability.

A recently studied micro-quasar in NGC5408 (Soria et al. 2006) shows up at a $B$ brightness $m_{B} \approx 23$ mag. A similar object could not have been detected in the abovementioned Gemini imagery, but should easily be observed with longer exposure times. The luminosity of this micro-quasar is $\log \left(L_{\mathrm{H} \alpha}\right) \simeq 36.18$ (Soria et al. 2006), which compares well with J133658.3-295105's luminosity of $\log \left(L_{\mathrm{H} \alpha}\right) \simeq 36.1 \pm 0.15$. Both sources are $\Delta \log \left(L_{\mathrm{H} \alpha}\right) \simeq 0.5$ fainter than the lower limit of the $\mathrm{H} \alpha$ luminosity function obtained from 17.797 extragalactic H II regions (Bradley et al. 2006). This strongly suggests that a mechanism that is different from that of ionizing stars is responsible for the $\mathrm{H} \alpha$ excitation in these objects.

As carried out by Laor \& Behar (2008) for radio-quiet quasars, we obtained the $R-X$ relation. To calculate $L_{R}=v L_{v}(6 \mathrm{~cm})$, we use, for RS28, $f(6 \mathrm{~cm})=0.87 \mathrm{mJy}$ (Maddox et al. 2006, 1998 observations) from the nearest date to that of the Chandra observation. We obtained $L_{R}=1.5 \times 10^{36} \mathrm{erg} \mathrm{s}^{-1}$. The Xray luminosity was calculated by the direct integration of the law presented in Table 1, yielding $L_{X}=1.1 \times 10^{38} \mathrm{erg} \mathrm{s}^{-1}$. Then, the $R-X$ relation is $\log \left(L_{R} / L_{X}(0.2-20 \mathrm{keV})\right) \simeq 0.01$, which is 2 orders of magnitude larger than the mean relation, $\log \left(L_{R} / L_{X}(0.2-20 \mathrm{keV})\right) \simeq 10^{-5}$, which fits cool stars, ULX, Seyfert nuclei, and radio-quiet quasars over an interval of more than 15 decades in X-ray luminosity (Laor \& Behar 2008). Although $L_{R}$ compares well with the interval of radio luminosities between ULX and Seyfert nuclei in Laor \& Behar (2008, Figure 3), the X-ray luminosity of J133658.3-295105 is approximately 2 orders of magnitude fainter than the faintest ULX source presented by the authors.

With respect to other space observations of M83, unfortunately, Hubble Space Telescope images do not cover the region of J133658.3-295105. Spitzer images at $5.8 \mu \mathrm{m}$ and $8 \mu \mathrm{m}$ bands with exposure times of $220 \mathrm{~s}$ and $210 \mathrm{~s}$ and brightness limits of $6.77 \mu \mathrm{Jy}$ and $12.95 \mu \mathrm{Jy}$ at $(\mathrm{S} / \mathrm{N})=3$, respectively, do not show an infrared counterpart at the object position.

\section{DISCUSSION}

The morphology of the radio emission distribution that protrudes along a line joining the $\mathrm{ON}$ of $\mathrm{M} 83$ and J133658.3-295105 suggests that the quasar-like object has been ejected from the ON, which is off-set with respect to the galaxy kinematical center. In that sense, it resembles the jet associated with the quasi-stellar object (QSO) PKS1127-14.5 which is also off-centered with respect to the QSO center (Siemiginowska et al. 2002). Nevertheless, the jet PKS1127-14.5 does not include an FRII radio source, as the one associated with J133658.3-295105.

An ejected $\mathrm{BH}$ may drag its accretion disk together and accrete interstellar matter during the transit through the bulge and the densest part of the disk (Volontieri 2007). If the lurking $\mathrm{BH}$ does not accrete fresh gas (argument suggested by the referee), it will be seen as an AGN as long as the accretion disk remains reasonably full. Radio-loud AGNs may occur at lower Eddington rates, perhaps implying that the disk is emptying out. For a lifetime of $10 \mathrm{Myr}$, the source moved at least $1.3 \mathrm{kpc}$ away from the nucleus, which implies a transverse velocity on the order of $100 \mathrm{~km} \mathrm{~s}^{-1}$. That velocity is comparable with the measured radial velocity for J133658.3-295105 and compatible with predicted kick-off ejection velocities, which run from a few hundred to a few thousand $\mathrm{km} \mathrm{s}^{-1}$ (Bonning et al. 2007; Blecha $\&$ Loeb 2006). The composed velocity is relatively small and the object would probably remain orbiting inside the galaxy until it decays back to the center, according to Fujita's model (Fujita 2009). In this case, the ejected BH will periodically accrete gas and its feeding will depend on its velocity as well as on the density and velocity dispersion of the interstellar medium that has been crossed by the object (Volontieri 2007).

Further insight can be obtained from Fujita (2009, and references therein). These models contemplate the ejection of an SMBH from the nucleus of a galaxy with bulge, disk, and halo components that are adequate for the Galaxy, with a circular velocity of $220 \mathrm{~km} \mathrm{~s}^{-1}$ at $R=7 \mathrm{kpc}$. Fujita assumes Chandrasekar dynamical friction, which makes the $\mathrm{BH}$ orbit decay back to the center. Most of Fujita's simulations assume that $20 \%$ of the disk is composed of gas, and that it is accreted into the SMBH according to the Bondi-Hoyle mechanism. The $\mathrm{X}$-ray luminosity of the SMBH is proportional to the accretion rate, to which an efficiency of about $10 \%$ is applied.

We take Fujita's models A1 and C3 and his Figures 7 and 9, respectively, as an example. The $\mathrm{SMBH}$ is launched from the nucleus, at $500 \mathrm{~km} \mathrm{~s}^{-1}$ and $700 \mathrm{~km} \mathrm{~s}^{-1}$ in the $(X, Z)$ plane, with an inclination of $30^{\circ}$ with respect to the galactic plane. Model A1 $\mathrm{BH}$ has $3 \times 10^{6} M_{\odot}$. The dynamical friction time $\left(t_{\mathrm{df}}\right)$, defined as the time that it takes the SMBH to decay to an orbit of a maximum radius of $10 \mathrm{pc}$, is $t_{\mathrm{df}}=0.63 \mathrm{Gyr}$. During the first 60 million years, the SMBH radiates $L_{X}=1 \times 10^{38 \pm 0.5} \mathrm{erg}$ $\mathrm{s}^{-1}$ at 14 and 44 Myr over periods of approximately 2-4 Myr. The relative velocity of the $\mathrm{SMBH}$ with respect to the disk is on the order of $250 \mathrm{~km} \mathrm{~s}^{-1}$ and its height with respect to the disk is around $0.1 \mathrm{kpc}$. Model C3 has an $M_{\mathrm{SMBH}}=3 \times 10^{7} M_{\odot}$ and $t_{\mathrm{df}}=3.7 \mathrm{Gyr}$. During the first $60 \mathrm{Myr}$, the $\mathrm{SMBH}$ radiates $L_{X}=1 \times 10^{38 \pm 0.5} \mathrm{erg} \mathrm{s}^{-1}$ at 10 and $50 \mathrm{Myr}$ during periods of approximately 10-20 Myr. In Model C3 the velocity with respect to the disk is between 250 and $300 \mathrm{~km} \mathrm{~s}^{-1}$, and its height with respect to the disk is between $0.7 \mathrm{kpc}$ and $1 \mathrm{kpc}$. The probability of observing the more massive $\mathrm{BH}$ is $\sim 5$ times higher, which point to Fujita's model $\mathrm{C} 3$ as more appropriated to describe the observed properties of J133658.3-295105.

\section{CONCLUDING REMARKS}

The Chandra X-rays spectrum of J133658.3-295105, a source projected on the body of M 83, presents Fe $\mathrm{K} \alpha$ line at $\mathrm{S} / \mathrm{N} \sim 2.5$ whose redshift $(z \ll 1)$ is compatible with that obtained from the $\mathrm{H} \alpha$ emission line $\left(620 \mathrm{~km} \mathrm{~s}^{-1}\right)$; both point to an object most likely located at the distance of M83.

Comparisons in the previous section (low X-ray luminosity, beaming, no surrounding nebulae) indicate that J133658.3-295105 is not likely to be a ULX, but still presents striking similarities to the micro-quasar in NGC5408. These similarities include the $\mathrm{H} \alpha$ luminosity $\left(\log \left(L_{\mathrm{H} \alpha}\right) \simeq\right.$ $36.1 \pm 0.15)$ and the very faint or absent $B$-band brightness $\left(m_{B}>22.5 \mathrm{mag}\right)$.

The range of masses that are suggested by Dottori et al. (2008b) on a very simple basis is around $10^{3}-10^{4}$ solar masses, but intermediate-mass BHs may have much higher luminosities (Farret et al. 2009). This makes one more inclined to accept the range of masses derived from the Fujita models for kickedoff BHs $\left(M_{\mathrm{BH}} \approx 10^{6}-10^{7} M_{\odot}\right)$, based on a more coherent theoretical scenario. Fujita's models with kick-off velocities of $300 \mathrm{~km} \mathrm{~s}^{-1}$ or smaller with respect to the galactic disk are more compatible with our results. 
Longer integration times would be necessary to achieve a higher $\mathrm{S} / \mathrm{N}$ to define the $\mathrm{Fe} \mathrm{K} \alpha$ line profile better and gain deeper insight into the physical characteristics of the J133658.3-295105 BH. This source may constitute the first detection of a kicked-off $\mathrm{BH}$ produced in a galactic nucleus.

The authors acknowledge the anonymous referee for helpful suggestions. J.F.A.C. is a research member of the Consejo Nacional de Investigaciones Científicas y Tecnológicas (CONICET). H.D., R.D., and D.M. acknowledge the support of MINCYT from Argentina and CAPES from Brazil. The Gemini Observatory is operated by the Association of Universities for Research in Astronomy, Inc., under a cooperative agreement with the NSF on behalf of the Gemini partnership, consisting of the NSF (USA), STFC (UK), NRC (Canada), ARC (Australia), MINCYT (Argentina), CNPq (Brazil) and CONICYT (Chile).

\section{REFERENCES}

Abraham, J. 2007, Science, 318, 938

Blecha, L., \& Loeb, A. 2006, MNRAS, 390, 1311

Bogdanovic', T., Eracleous, M, \& Sigurdsson, S. 2009, ApJ, 697, 288

Bonning, E., Shields, G. A., \& Salviander, S. 2007, ApJ, 666, L13

Boroson, T. A., \& Lauer, T. R. 2009, Nature, 458, 53

Bradley, T. R., Knapen, J. H., Beckman, J. E., \& Folkes, S. L. 2006, A\&A, 459, 13

Brickhouse, N. S., Desai, P., Hoogerwerf, R., Liedahl, D. A., \& Smith, R. K. 2005, in AIP Conf. Proc. 774, X-ray Diagnostics of Astrophysical Plasmas: Theory, Experiment, and Observation, ed. R. K. Smith (Melville, NY: AIP), 405
Cash, W. 1979, ApJ, 228, 939

Cowan, J. J., Roberts, D. A., \& Branch, D. 1994, ApJ, 434, 128

Diaz, R. J., Dottori, H., Aguero, M. P., Mediavilla, E., Rodrigues, I., \& Mast, D. 2006, ApJ, 652, 1122

Dottori, H., Diaz, R., Agüero, M. P., Mast, D., \& Rodrigues, I. 2008a, in IAU Symp. 245, Formation and Evolution of Galaxy Bulges, ed. M. Bureau, E. Athanassoula, \& B. Barbuy (Cambridge: Cambridge Univ. Press), 297

Dottori, H., Diaz, R., \& Mast, D. 2008b, AJ, 136, 2468

Eckart, M., Stern, D, Helfand, D., Harrison, F., Mao, P., \& Yost, S. 2006, ApJS, 165,19

Elmegreen, D., Chromey, F., \& Warren, A. 1998, AJ, 116, 2834

Farrett, S., et al. 2009, Nature, 460, 73

Ferrarese, L., \& Merrit, D. 2000, ApJ, 539, L9

Fujita, Y. 2009, ApJ, 691, 1050

Gebhardt, K., et al. 2000, ApJ, 539, L13

Houck, J. C., \& Denicola, L. A. 2000, in ASP Conf. Proc. 216, Astronomical Data Analysis Software and Systems IX, ed. N. Manset, C. Veillet, \& D. Crabtree (San Francisco, CA: ASP), 591

Iwasawa, M., Funato, Y., \& Makino, J. 2006, ApJ, 651, 1059

Karachentsev, I. D., et al. 2002, A\&A, 385, 21

Kembhavi, A. K., \& Narlikar, J. V. 1999, Quasars and Active Galactic Nuclei (Cambridge: Cambridge Univ. Press)

Laor, A., \& Behar, E. 2008, MNRAS, 390, 847

Maddox, L., et al. 2006, AJ, 132, 310

Mushotzky, R. 2004, Prog. Theor. Phys. Suppl., 155, 27

Okamoto, T., Nemmen, R., \& Bower, R. G. 2008, MNRAS, 385, 161

Siemiginowska, A., et al. 2002, ApJ, 570, 543

Soria, R., \& Wu, K. 2003, A\&A, 410, 53

Soria, R., et al. 2006, MNRAS, 368, 1527

Soria, R., et al. 2009, arXiv:0912.2732v1

Stockdale, C. J., et al. 2001, BAAS, 199, 1902

Thatte, N., Tecza, M., \& Genzel, R. 2000, A\&A, 364, L47

Volonteri, M. 2007, ApJ, 663, L5 